\title{
CONSTRUÇÃO DE UM QUADRO-CRISTAL PARA APLICAÇÃO EM AMBIENTES HÍBRIDOS DE ENSINO TECNOLÓGICO
}

\author{
Fernando Luiz Conceição Alves, Secretaria de Educação/GO, \\ fernandoluiz.alves@seduc.go.gov.br, \\ ORCID: https://orcid.org/0000-0002-6381-2917 \\ Thiago Viana de Freitas, Instituto Federal de Educação, Ciência e Tecnologia de \\ Brasília, thiago.freitas@ifb.edu.br, \\ ORCID: https://orcid.org/0000-0002-1842-4982 \\ José Giovanni Leite de Brito, Instituto Federal de Educação, Ciência e Tecnologia de \\ Brasília, jose.brito@ifb.edu.br, \\ ORCID: https://orcid.org/0000-0002-4080-4885
}

\begin{abstract}
RESUMO: Os estudantes modernos necessitam de estratégias de ensino criativas, engajadoras, e que sejam capazes de minimizar as distrações. Neste contexto, as metodologias ativas com salas de aula invertidas apoiadas em videoaulas se destacam, pois permitem que os alunos façam o autogerenciamento da duração e do ritmo de aprendizagem. Pensando em contribuir para a melhoria do ensino em uma instituição de ensino profissionalizante de Brasília, decidimos construir uma infraestrutura para gravação de videoaulas onde o instrutor fica frente a frente com a câmera enquanto este realiza anotações com canetas luminescentes em uma superfície transparente: o quadrocristal. Neste artigo compartilhamos nossas experiências durante a construção do primeiro estúdio de lightboard do Distrito Federal, bem como discutimos as boas práticas de uso da ferramenta e mostramos algumas possibilidades didáticas de como ela pode ser explorada no Ensino de Química.
\end{abstract}

Palavras-chave: ensino híbrido, aula invertida, ensino de química, quadro-cristal, OBS

\section{CONSTRUCTION OF A LIGHTBOARD FOR APPLICATION IN HYBRID ENVIRONMENTS OF PROFESSIONAL TEACHING}

\begin{abstract}
Modern students have the need for creative and engaging teaching strategies capable of minimizing distractions. In this context, active methodologies with video-based flipped classrooms stand out, as they allow students to manage their own learning duration and pace. With the aim to contribute with the teaching betterment of a Brazilian professional school we have decided to build an infrastructure for recording videos where the instructor faces the camera while writes with luminescent markers on a transparent surface: the lightboard. In this paper, we share our experiences during the construction of the first lightboard studio at Federal District, as well as we discuss the good practices of using the tool and show some teaching possibilities of how it can be explored in Chemistry Teaching.
\end{abstract}

Keywords: hybrid teaching, flipped classroom, chemistry teaching, lightboard, OBS

\section{INTRODUÇÃO}

Os avanços tecnológicos modernos têm permitido e estimulado educadores ao redor do planeta a adotarem novos métodos e estratégias de ensino. Igualmente, o perfil dos estudantes digitais e a hiper conectividade que eles possuem com as tecnologias emergentes originam sobre os sistemas educacionais uma forte demanda por resultados V. $19 \mathrm{~N}^{\circ} 1$, julho, 2021 DOI: https://doi.org/10.22456/1679-1916.118528

RENOTE 
práticos relacionados à uma educação engajadora. Além disso, estudos recentes apontam que os estudantes imersos na contemporaneidade necessitam de estratégias de ensino mais criativas e instigantes, pois eles são bem mais facilmente distraídos (Bishop e Verleger, 2013).

Em face desses novos desafios, surgiu um novo paradigma para o ensino que vem ganhando grande atenção nos últimos anos, e o qual é conhecido como sala de aula invertida (Pienta, 2016). A sala de aula invertida é um conceito que encontra fundamentos no ensino híbrido e, neste modelo, os alunos estudam os conteúdos e materiais disponibilizados pelo professor previamente ao encontro presencial (Bergmann e Sams, 2016). Após a exploração independente dos conteúdos, os alunos partem para uma etapa posterior, dentro da sala de aula, de aprofundamento de conceitos, desenvolvimento de atividades, esclarecimento de dúvidas e realização de atividades avaliativas. A aprendizagem é então consequência dessa interatividade reversa (Seery, 2015).

Em uma sala de aula invertida típica, os estudantes aprendem e estudam fora da sala de aula, em seu próprio ritmo, através de aulas que exploram materiais interativos que podem ser vídeos pré-gravados, podcasts, textos, jogos eletrônicos, slides, e-books, ou qualquer outro material disponível que possa servir de meio para uma aprendizagem dinâmica e autônoma. Na etapa posterior é onde os educadores encontram terreno para identificar e mitigar as dificuldades enfrentadas pelos estudantes. É desta forma que o aprendizado acaba sendo incrementado (Fulton, 2012).

Muito comuns em metodologias com aulas invertidas, aulas narradas com slides de Powerpoint são muito úteis para o aprendizado dos estudantes, pois os vídeos podem ser acessados em plataformas variadas, em alguns casos sem a necessidade de acesso à rede mundial de computadores. Além disso, opostamente às preleções numa sala de aula tradicional, os estudantes podem assistir os vídeos quantas vezes desejarem, até o entendimento pleno do conteúdo assistido (Júnior, 2019).

Quando se usam vídeos nas metodologias de aula invertida, por exemplo, as aulas pré-gravadas exibem o apresentador lecionando sua matéria em um quadro-branco ou narrando projeções de slides. Avaliando por este aspecto, os estudantes aprenderão ativamente, pois eles terão a liberdade para explorar a aula e os materiais associados a ela, de tal forma que o ritmo de cada um e a duração para completar o aprendizado serão respeitados. Ainda assim, a duração dos vídeos e os conteúdos exibidos podem ser objetos causadores de distração, fatores estes que geram desafios para a eficácia da ferramenta (Ruiperez-Valiente et. al., 2015). Dentre as principais reivindicações, estudantes apontam que muitas aulas são a mera leitura dos slides feita pelo professor, o que gera o sentimento de que os conteúdos poderiam ter sido aprendidos sem a necessidade de assistir os vídeos. Adicionalmente, os estudantes possuem uma preferência por vídeos mais curtos e sucintos no estilo dos vídeos da "Khan Academy", os quais apresentam instrutores narrando os conteúdos que aparecem em um quadro-negro eletrônico (Ruiperez-Valiente et. al., 2015). Essas preferências foram confirmadas por meio de estudos recentes feitos por Guo, Kim e Rubin (2014), que concluíram que vídeos mais curtos, e com a presença do rosto do apresentador tornam o conteúdo mais estimulante.

Para contornar algumas das barreiras anteriormente relatadas acerca da distração ocasionadas pelo uso de videoaulas, universidades estrangeiras e algumas instituições de educação brasileiras estão implementando uma ferramenta de gravação de vídeos que permite ao instrutor ficar frente a frente com a câmera enquanto este realiza anotações em uma superfície transparente. Nesta técnica, internacionalmente conhecida como lightboard, fitas de LED são estrategicamente posicionadas nas bordas de um quadro de vidro e quando acionadas fazem brilhar as substâncias fluorescentes presentes em canetas 
de neon. Esta configuração permite aos instrutores ilustrar as suas aulas sem o bloqueio natural feito pelo corpo, e sem que o apresentador vire as costas para o seu público.

O lightboard, ou quadro-cristal ${ }^{\mathrm{a}}$, é um hardware de código aberto, e uma técnica de gravação de videoaulas criada pelo professor de engenharia Michael A. Peshkin, da Northwestern University, localizada no estado de Illinois, nos Estados Unidos (Peshkin, 2020). Como já relatado, a técnica consiste em uma lousa de vidro ou acrílico na qual o professor faz as anotações enquanto explica o conteúdo diante de uma câmera. O quadrocristal permite a produção de vídeos em alta qualidade, e a sua principal característica é o fato de que o apresentador fica de frente para a audiência ao mesmo tempo em que ele grava a sua instrução da maneira como ele já está acostumado. Estas particularidades conferem às aulas com quadro-cristal diversas vantagens sobre as aulas convencionais em quadro-negro e sobre os vídeos convencionais, tais como uma maior motivação e um maior interesse nas aulas por parte dos estudantes. Com a técnica, o trabalho de edição de videoaulas é facilitado, e muitos outros recursos, tais como slides, gráficos, objetos e até vídeos, podem ser incorporados à tela (Mccorkle e Whitener, 2020). Isto é particularmente útil para instrutores de disciplinas das áreas de ciências exatas, que geralmente encontram a necessidade de complementar as explicações verbais com equações, esquemas, figuras e diagramas.

Ainda não existem versões comerciais disponíveis para a ferramenta no Brasil, entretanto muitas instituições - majoritariamente estrangeiras - estão começando a desenvolver versões melhoradas, geralmente baseadas no modelo lightboard padrão desenvolvido pelo Prof. Peshkin, e com customizações orientadas pelas necessidades de cada instituição. Em instituições públicas do Brasil, a tecnologia ainda é extremamente incipiente, havendo relatos de seu uso apenas na USP por professores do Departamento de Engenharia de Estruturas e Geotécnica da Escola Politécnica (Meneghetti et. al., 2018) e no Colégio Técnico da UFMG (COLTEC), por professores do Curso Técnico em Química (Mateus et. al., 2018).

Inspirados por esse estado-da-arte, apresentamos neste artigo nossas experiências durante as etapas de criação do primeiro laboratório de produção de videoaulas em lightboard no Distrito Federal. Nosso grupo possui um projeto em andamento que visa produzir, com esta ferramenta, material instrucional em vídeo para aplicação em ambientes híbridos de ensino. Assumindo o grande potencial do lightboard para incrementar o engajamento de estudantes, pretendemos, desta forma, contribuir para a melhoria do ensino profissionalizante no Brasil.

\section{CONSTRUÇÃO DO QUADRO-CRISTAL}

O primeiro estúdio de quadro-cristal do Distrito Federal foi montado de forma conjugada ao Laboratório de Ensino de Química do Instituto Federal de Brasília/Campus Gama. O primeiro passo para a edificação do estúdio foi a construção da estrutura metálica que serviu de suporte para o quadro de vidro. Para isso, realizamos algumas modificações no design proposto pela empresa americana Revolution Lightboards, sendo então contratada mão-de-obra especializada que construiu a estrutura em metalon 40 x 30 $\mathrm{mm}$, na chapa 18 , com zarcão cinza, e dimensões personalizadas $(2,00 \mathrm{~m}$ de comprimento x 2,00 $\mathrm{m}$ de altura $\mathrm{x} 0,90 \mathrm{~m}$ de profundidade). As principais modificações introduzidas no projeto foram duas hastes laterais que serviram como suporte para os bastões de LED, 2 suportes para marcadores e apagadores, e 4 rodas de silicone para auxiliar no transporte do conjunto.

A seguir, adquirimos no comércio varejista brasiliense uma placa de vidro temperado Extra Clear com $10 \mathrm{~mm}$ de espessura, 2,10 $\mathrm{m}$ de comprimento e 1,05 $\mathrm{m}$ de 
altura. $\mathrm{O}$ vidro foi instalado na estrutura metálica por meio de 4 furos localizados a $5 \mathrm{~cm}$ de suas bordas laterais, e foi preso à estrutura metálica usando-se 4 prolongadores de alumínio de $25 \mathrm{~mm}$ x $50 \mathrm{~mm}$. A realização dos furos no vidro - com diâmetros iguais a 5 $\mathrm{mm}$ - foi executada pela empresa especializada antes do temperamento do vidro e levou em consideração o peso e a margem de segurança necessários para não haver o risco de trincamento do vidro.

No próximo passo, foi realizada a instalação da fita LED nas bordas do quadrocristal. Para isso, foram utilizados perfis extrudados de alumínio do tipo PU com canais de $10 \mathrm{~mm}$ de espessura, e nas dimensões perimetrais da placa de vidro. As 4 peças foram personalizadas por profissional especializado, e foram conectadas com design exterior a ângulos de $45^{\circ}$. A seguir, uma fita LED branca de $5 \mathrm{~m}$ (240 leds) e 280 watts de potência foi inserida nos canais de $10 \mathrm{~mm}$ do perfil PU, sendo os canais previamente preenchidos com pasta térmica para dissipar a grande quantidade de calor produzido pela fita, e este conjunto foi então posicionado nas bordas do vidro Extra Clear. O acabamento para unir as peças independentes foi feito com fita dupla face $3 \mathrm{M}$ e fita isolante. $\mathrm{O}$ acionamento da fita LED se deu por alimentação externa via interruptor, sendo este modo de trabalho alcançado com fonte chaveada de 10 A e 120 watts de potência, montada dentro de caixa de segurança customizada e acoplada à estrutura metálica.

Quanto ao sistema de iluminação, foi implementada a técnica profissional conhecida como iluminação de 3 pontos, que é um sistema formado por 3 fontes de luz, direcionadas para a pessoa a ser gravada. Em nosso sistema, as luzes frontal e de preenchimento foram alcançadas por meio da instalação de 3 luminárias LED de $36 \mathrm{~W}$ e brilho lúmen de $6500 \mathrm{~K}$ cada. Estas foram posicionadas a $45^{\circ}$ das hastes laterais direita e esquerda da moldura metálica (onde foram instaladas 2 luminárias), sendo a terceira luminária posicionada na porção superior central da moldura. Um refletor LED móvel de $200 \mathrm{~W}$ A foi utilizado como luz de fundo (backlight).

Além do vidro de aprendizagem e do sistema de iluminação, o layout do estúdio contou ainda com um painel backdrop preto de tecido Oxford, sendo empregada uma peça com 3 metros de comprimento por 2 metros de altura, localizada a 1,5 $\mathrm{m}$ do quadrocristal. Nas extremidades laterais do tecido foram confeccionadas duas barras para acoplamento das hastes móveis de sustentação feitas com cano PVC.

Dentre os principais componentes eletrônicos usados na montagem do estúdio destacam-se uma câmera DSLR profissional, sendo utilizada uma Canon EOS Rebel T6i apoiada em tripé simples. A estação de trabalho utilizada durante as gravações foi um laptop Dell Inspiron 155000 dotado com sistema operacional Windows 10, processador Intel Core i3 $6^{\text {a }}$ Geração, e 4 GB de DDR2. Ainda, foi utilizado como monitor de referência uma TV Panasonic de 49" localizada a 1,50 $\mathrm{m}$ do vidro de aprendizagem e conectada diretamente à estação de trabalho por meio de cabo HDMI. Para a captação da voz do apresentador foi utilizado um microfone de lapela com fio, conectado diretamente na entrada de áudio do laptop Dell. Marcadores luminescentes de cores variadas da marca Windows Marker foram utilizados para a interação no quadro-cristal.

Para a captação e enquadramento das cenas, foi utilizada a ferramenta Live Remote View do software EOS Utility, o qual é um assistente dos produtos Canon ${ }^{\circledR}$ e que pode ser baixado gratuitamente em qualquer sistema operacional. Por fim, os vídeos foram exportados em formato $\mathrm{mp} 4$ com qualidade HD (1080 x 720), gravados a 25 FPS modo PAL, sendo as gravações gerenciadas com o Open Broadcaster Software ${ }^{\circledR}$, um aplicativo gratuito e de código aberto para gravação de vídeo e transmissão em tempo real. 


\section{RESULTADOS E DISCUSSÃO}

$\mathrm{Na}$ Figura 1 são exibidas as principais características do quadro-cristal desenvolvido neste trabalho. A construção do protótipo foi viabilizada por meio do programa institucional da Pró-Reitoria de Pesquisa e Inovação do Instituto Federal de Brasília, denominado "Fábrica de Ideias Inovadoras". Este programa tem como objetivo estimular proponentes a apresentarem ideias potencialmente inovadoras, estabelecendo contato com a cultura e os procedimentos relacionados à inovação tecnológica. Nesse sentido, nosso grupo de pesquisa angariou recursos para custear o desenvolvimento do laboratório e adquirir seus principais elementos.
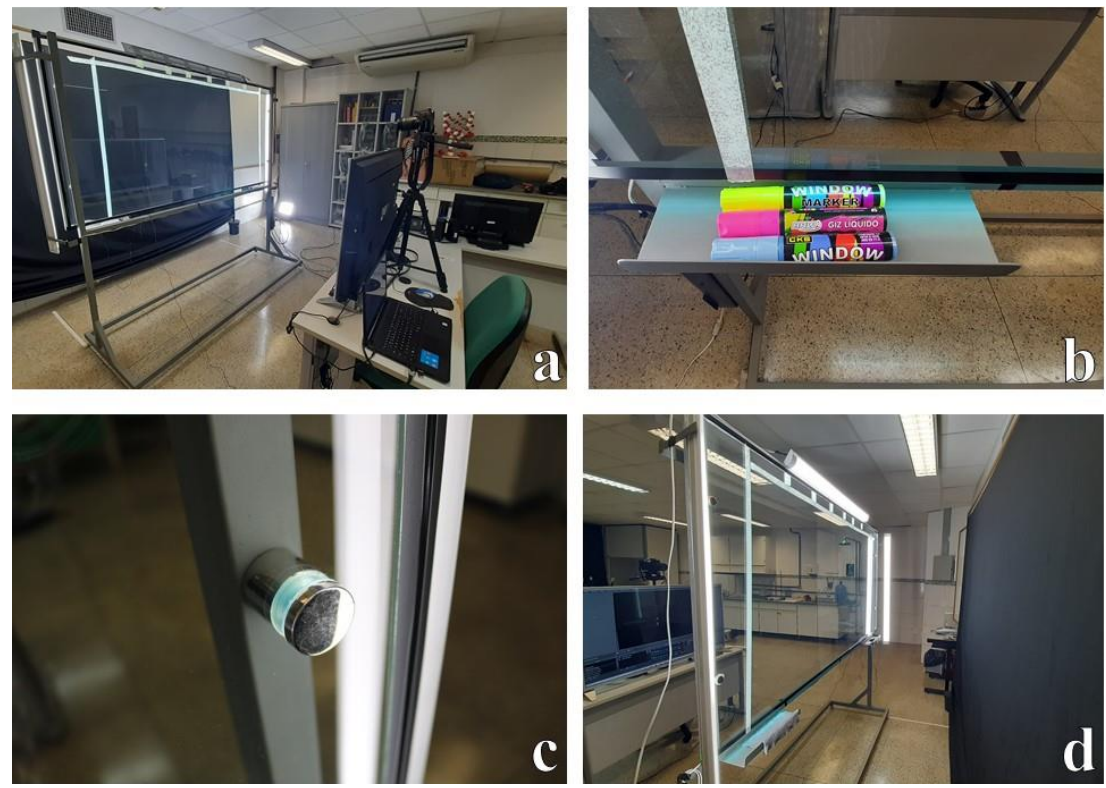

Figura 1 - Principais características do estúdio de quadro-cristal abordado neste trabalho. a) quadrocristal, suporte, e estação de trabalho em destaque; b) suporte metálico para guardar os materiais didáticos. c) prolongador de fixação do vidro no stand; d) bastões laterais de LED e backdrop.

A construção e montagem de um quadro-cristal pode ocorrer de forma relativamente rápida, com moderado labor e baixo orçamento. No caso aqui descrito, entre o design do stand metálico, sua construção, e a fabricação e instalação do vidro e das fitas LED, passaram-se aproximadamente 45 dias, e todo o processo contou com a participação de apenas 4 colaboradores especializados a um custo total aproximado de $\mathrm{R} \$$ 3.400,00 (três mil e quatrocentos reais), embora exista a possibilidade de criação de laboratórios de videoaulas em lightboard com bem menos recursos. Mccorkle e Whitener (2020), por exemplo, abordam a construção de um lightboard com materiais de baixo custo (moldura de madeira e placa de acrílico), mas nós optamos por uma configuração semiprofissional que fosse capaz de atender à demanda de um campus com vocação profissionalizante e que oferta 8 cursos das mais variadas modalidades de ensino.

Vale destacar aqui a maior dificuldade enfrentada durante o desenvolvimento do laboratório: a aquisição do vidro Extra Clear. O desafio foi colossal, especialmente por conta da retração de oferta imposta pela pandemia de coronavírus, bem como pelas especificidades do material, que o tornam de difícil aquisição. Quando inicialmente orçada em novembro de 2019, a peça de vidro nas dimensões $2,0 \mathrm{~m}$ x 1,0 m x $10 \mathrm{~mm}$ estava custando aproximadamente $\mathrm{R} \$ 800,00$ (oitocentos reais). No momento real da aquisição (setembro/2020) as lojas especializadas estavam comercializando o mesmo V. $19 \mathrm{~N}^{\mathrm{o}} 1$, julho, 2021 RENOTE 
item por, no mínimo, o dobro desse valor. Ainda assim, acreditamos que essa dificuldade não seria tão grande nas regiões onde os vidros de altíssima transparência são fabricados - regiões Sul e Sudeste.

O quadro de vidro é iluminado internamente pela fita LED branca de alta intensidade que entra pelas bordas do material vítreo. A luz atinge as partes internas do vidro pelo fenômeno de reflexão interna total, e é isto o que garante a iluminação dos marcadores, característica mais marcante na técnica de lightboard. Em testes que não exploraremos neste artigo observamos que vidros comuns - com bordas na cor verde musgo - afetam drasticamente essa iluminação interna. Por conta dos altos teores de sais de ferro e a consequente coloração escura dos vidros comuns, pouca luz consegue chegar ao centro. Nesse sentido, o vidro Extra Clear - que possui coloração azul claro nas bordas - se mostrou bastante adequado para os objetivos deste trabalho. Pudemos observar ainda que a espessura de $10 \mathrm{~mm}$ empregada ocasiona leves trepidações quando o apresentador escreve nas regiões centrais do quadro, possivelmente devido ao seu tamanho.

A moldura do nosso quadro é fixa, portanto, recomendada para gravação em plano médio, e para usuários com estatura entre 1,60 - 1,80 m. No estúdio, o instrutor dispõe de uma área de $1,80 \mathrm{~m}^{2}$ para transitar e se movimentar durante as aulas. É importante ponderar que dispositivos mais avançados e sofisticados, dotados com sistemas de elevação do quadro de vidro (Mccorkle e Whitener, 2020), embora disponíveis a um custo elevado, podem favorecer a participação de instrutores das mais variadas alturas, inclusive aqueles com mobilidade reduzida. Sendo assim, já é uma perspectiva futura para o nosso grupo de pesquisa incrementar o estúdio com essa tecnologia.

Nas videoaulas em quadro-cristal o apresentador interage face-a-face com os espectadores e escreve textos e esquemas que parecem estar ao contrário, mas na verdade estão orientados apropriadamente para o espectador. Uma vez que a câmera está de frente para o vidro de aprendizagem, o vídeo gravado fica ao contrário, sendo necessário uma etapa de edição para reverter a imagem. A reversão da imagem pode ser feita ainda gravando-se a imagem especular das aulas - embora isto acrescente custos adicionais ao projeto (Schweiker et. al., 2020). Em nosso caso, a reversão da imagem foi feita sem custos e eliminando-se a etapa de pós-produção, bastando apenas selecionar a cena e aplicar no programa OBS o comando "Transformar $\rightarrow$ Inverter na horizontal". Adicionalmente, implementamos em nosso estúdio um monitor de confidência, uma TV de 49 polegadas localizada a 1,50 $\mathrm{m}$ do vidro, e cujo objetivo principal é propiciar ao instrutor a capacidade de monitoração das suas anotações em tempo real. Um problema adicional que surge com a instalação desse monitor de referência é o surgimento de seu reflexo no vidro, mas que foi facilmente eliminado quando utilizamos um filtro polarizador circular de $58 \mathrm{~mm}$ da Greika acoplado na lente $18-55 \mathrm{~mm}$ da câmera Canon.

As aulas em quadro-cristal são uma mescla das preleções tradicionais em quadro branco com os estímulos oferecidos pelo quadro negro digital encontrado nas aulas do Khan Academy. Nesse sentido, para atingir o efeito de fundo negro nosso estúdio dispõe de um backdrop preto feito de tecido Oxford que está localizado a 2,50 m da câmera e fica atrás do apresentador. Ainda que sejam utilizados tecidos especiais absorvedores de luz - como o tecido Muslin ou TNT fotográfico - existe a necessidade de controle de brilho das cenas. Nós fizemos isso de duas maneiras: controlando a sensibilidade ISO do dispositivo de gravação e fazendo correção de brilho usando os plugins nativos do OBS. $\mathrm{Na}$ câmera, o ajuste ideal foi encontrado quando o dispositivo operou no modo de gravação manual, sendo fixadas a sensibilidade ISO em 200, a velocidade do obturador em 1/30, e a abertura do diafragma em F4.0. O ajuste fino de brilho foi feito no OBS, selecionando-se a fonte e seguindo o comando Filtros $\rightarrow$ Filtros de efeito $\rightarrow$ Correção de Cor. Após etapa de otimização, as propriedades Gama, Contraste e Brilho foram 
estabelecidas para $-0,50,0,10$, e 0,08 , respectivamente. Com estas configurações foi possível eliminar os reflexos indesejados do vidro e do monitor de referência, proporcionando ainda uma iluminação apropriada para destacar a silhueta do apresentador (Figura 2).
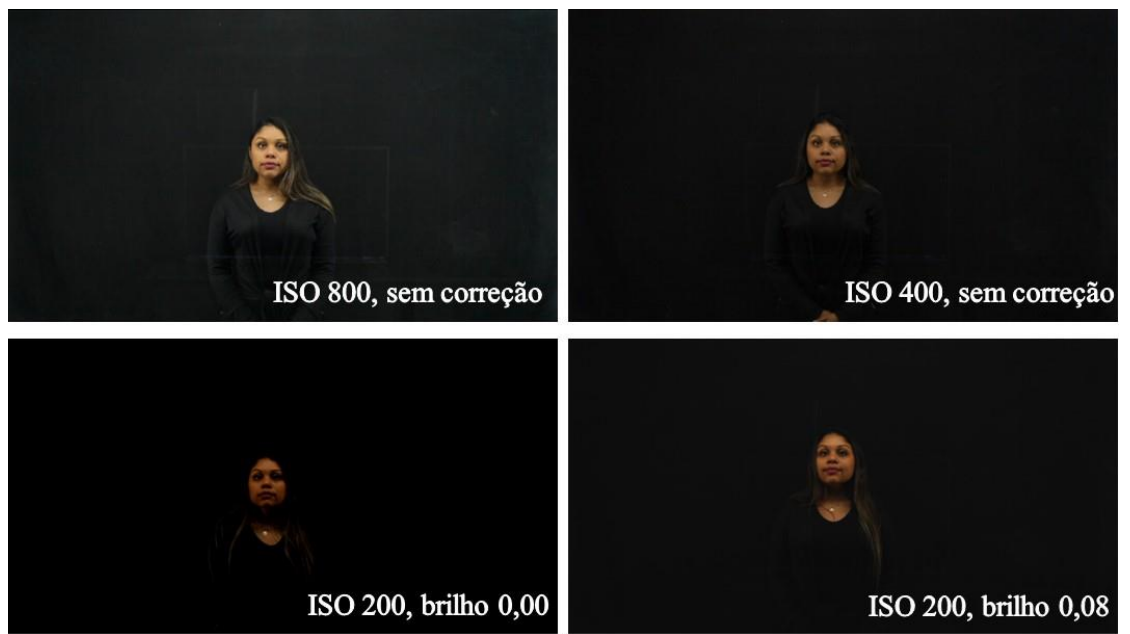

Figura 2 - O brilho das cenas foi controlado através de uma combinação da sensibilidade ISO da câmera com a aplicação de filtros corretivos usando os plugins nativos do OBS. Com o ISO 200 e correção de brilho em 0,08 encontramos o ajuste ideal para minimizar os reflexos indesejados.

Para as gravações utilizado o OBS, na aba de configurações do software, o modo de saída foi definido como 'Avançado' e o Tipo de saída definido como 'Padrão', sendo utilizado o codificador QuickSynch H.264 da máquina Dell Inspiron. A seguir, em testes preliminares observamos a ocorrência de vídeos com muitos "lags", que são atrasos experimentados na comunicação entre o software e a placa do laptop, originando vídeos com falhas, e sem sincronia entre o áudio e o vídeo. Diante disso, decidimos conduzir um estudo metodológico de otimização dos parâmetros de gravação para assim gerar vídeos com os melhores resultados de resolução e fluidez. Para isso, as seguintes propriedades foram congeladas no OBS: Uso alvo em 'quality'; Perfil de gravação em 'high'; Intervalo de Keyframe em 10; Latência como 'ultra-low'; B frames em ' 3 '. Os demais parâmetros (Controle da Taxa de Bits, Taxa de Bits e Taxa de Bits Máxima) foram avaliados, e o resumo da otimização encontra-se disponível na Tabela 1.

Tabela 1 - Avaliação da ocorrência de lags por meio da otimização da taxa de bits.

\begin{tabular}{ccccc}
\hline Entrada & Controle da taxa de bits & Taxa de bits & Taxa de bits máxima & Presença de lag \\
\hline 1 & CBR & $50000 \mathrm{kbps}$ & $5000 \mathrm{kbps}$ & sim \\
2 & CBR & $70000 \mathrm{kbps}$ & $7000 \mathrm{kbps}$ & não \\
3 & CBR & $100000 \mathrm{kbps}$ & $10000 \mathrm{kbps}$ & não \\
4 & VBR & $50000 \mathrm{kbps}$ & $5000 \mathrm{kbps}$ & sim \\
5 & VBR & $70000 \mathrm{kbps}$ & $7000 \mathrm{kbps}$ & não \\
6 & VBR & $100000 \mathrm{kbps}$ & $10000 \mathrm{kbps}$ & não \\
7 & VBR & $120000 \mathrm{kbps}$ & $12000 \mathrm{kbps}$ & não \\
8 & VCM & $50000 \mathrm{kbps}$ & $5000 \mathrm{kbps}$ & sim \\
9 & VCM & $70000 \mathrm{kbps}$ & $7000 \mathrm{kbps}$ & não \\
10 & VCM & $100000 \mathrm{kbps}$ & $10000 \mathrm{kbps}$ & não \\
\hline
\end{tabular}

Conforme pode ser visualizado na Tabela 1, nós definimos a taxa de bits máxima como $10 \%$ da taxa de bits, e observamos ainda que taxas de bits a partir de $70000 \mathrm{kpbs}$ suprimem a ocorrência de lags, mas têm como consequência a geração de vídeos (duração aproximada de 2 minutos) com tamanho superior a 800 megabytes, o que gera dificuldades relativas ao armazenamento, consumo de memória, e compartilhamento. A V. $19 \mathrm{~N}^{\mathrm{o}} 1$, julho, 2021 RENOTE 
razão bits:qualidade ideal foi obtida quando a taxa de bits teve o VBR como controlador, onde passamos a utilizar taxa de $100000 \mathrm{kbps}$ e a taxa de bits máxima igual a $10000 \mathrm{kpbs}$ (Entrada 6, Tabela 1). Nessas condições, vídeos-testes com duração de aproximadamente 2 minutos apresentaram ótima resolução e fluidez, além de ficarem com tamanhos menores que 100 megabytes.

Com relação à captação da voz do instrutor, de início percebemos que apenas o áudio captado pela câmera não teria qualidade satisfatória, uma vez que o vidro fica no caminho entre a câmera e a fonte sonora (voz). Diante disso, implementamos um sistema de captação de som semiprofissional, sendo utilizado um microfone de lapela com fío conectado diretamente na porta P2 do notebook Dell. Cabe ressaltar que nesta fase conseguimos eliminar mais uma etapa de edição onde, geralmente, áudio e vídeo são gravados separadamente e depois sincronizados em pós-produção. Em nosso caso, foi possível otimizar os parâmetros de áudio dentro do OBS, o qual exportava os vídeos já contendo o áudio devidamente sincronizado e com ótima qualidade. Outra vantagem oferecida pelo software é a possibilidade de uso de plugins para a eliminação de ruídos e para a equalização do áudio.

Para obter anotações com o efeito característico das aulas em lightboard é necessário o uso de canetas luminescentes, as quais contém substâncias fluorescentes que brilham quando são atingidas pela incidência das luzes LED. Em nosso estúdio foram utilizadas canetas da marca Window Marker com pontas medindo 8 x $15 \mathrm{~mm}$, laváveis com água. A escolha dessas canetas foi intencional, uma vez que elas propiciam ao instrutor escrever traçados finos e grossos. Um grande desafio encontrado aqui diz respeito à limpeza do quadro de vidro. Embora seja possível o uso de papéis absorvedores, tivemos grande dificuldade para remover os excessos da tinta fluorescente com esse material. Tentamos ainda empregar líquidos limpa-vidros para auxiliar na remoção da tinta fluorescente, mas nossa experiência permite recomendar a limpeza apenas com panos de microfibra úmidos, seguida por secagem com outro pano de microfibra seco.

Sabe-se que alguns conceitos das Ciências Exatas geram dificuldades de compreensão nos estudantes, especialmente nas disciplinas Química, Física e Matemática (Santos, et. al., 2013). Vídeos estimulantes e motivadores produzidos em quadro-cristal poderiam permitir a esses aprendizes rever tais conceitos tantas vezes quanto necessárias até o seu total entendimento. Para a disciplina Química, por exemplo, conceitos envolvendo cálculos estequiométricos, equilíbrios químicos, termoquímica, e química orgânica, envolvem temas com potencial para uma melhor compreensão, caso disponibilizados em aulas no quadro-cristal. Além disso, aulas em lightboard já demonstraram ter potencial para incrementar o engajamento de estudantes em aulas de Química (Fung, 2017).

Inspirados pelo potencial engajador da ferramenta lightboard, nosso grupo decidiu produzir conteúdo instrucional de Química para ilustrar as possibilidades de aplicação da tecnologia. Para a seleção dos conteúdos, levamos em consideração as principais demandas discentes de uma escola profissionalizante de Brasília, tais como: maior dificuldade de aprendizado; menores taxas de rendimento; menor motivação. Nesse sentido, produzimos um lote de videoaulas em quadro-cristal abordando os temas estequiometria, interações intermoleculares e mecanismos de reações orgânicas. As aulas foram elaboradas e otimizadas por meio de roteirização, sendo explorada a superimposição de elementos gráficos como slides, vídeos, animações e imagens. Os materiais não passaram por etapa de pós-produção, e cada aula teve, no máximo, 10 minutos.

Na Figura 3, ilustramos algumas possibilidades didáticas de aplicação das aulas em lightboard no Ensino de Química. Vale destacar que em sistemas mais avançados a 
inclusão de elementos gráficos é bastante dispendiosa, necessitando, por exemplo, do uso de switchers e mesas de corte (Peshkin, 2020). Aqui, mais uma vez, conseguimos atingir eficiência por meio do planejamento racional e roteirização das aulas. O próximo passo de nossa pesquisa é aplicar os materiais produzidos no ensino profissionalizante e aferir a sua capacidade de engajamento. Isto será feito usando-se as videoaulas em lightboard nas trilhas de aprendizagem que exploram metodologias ativas com sala de aula invertida, especialmente aquelas hospedadas em ambientes virtuais de ensino.
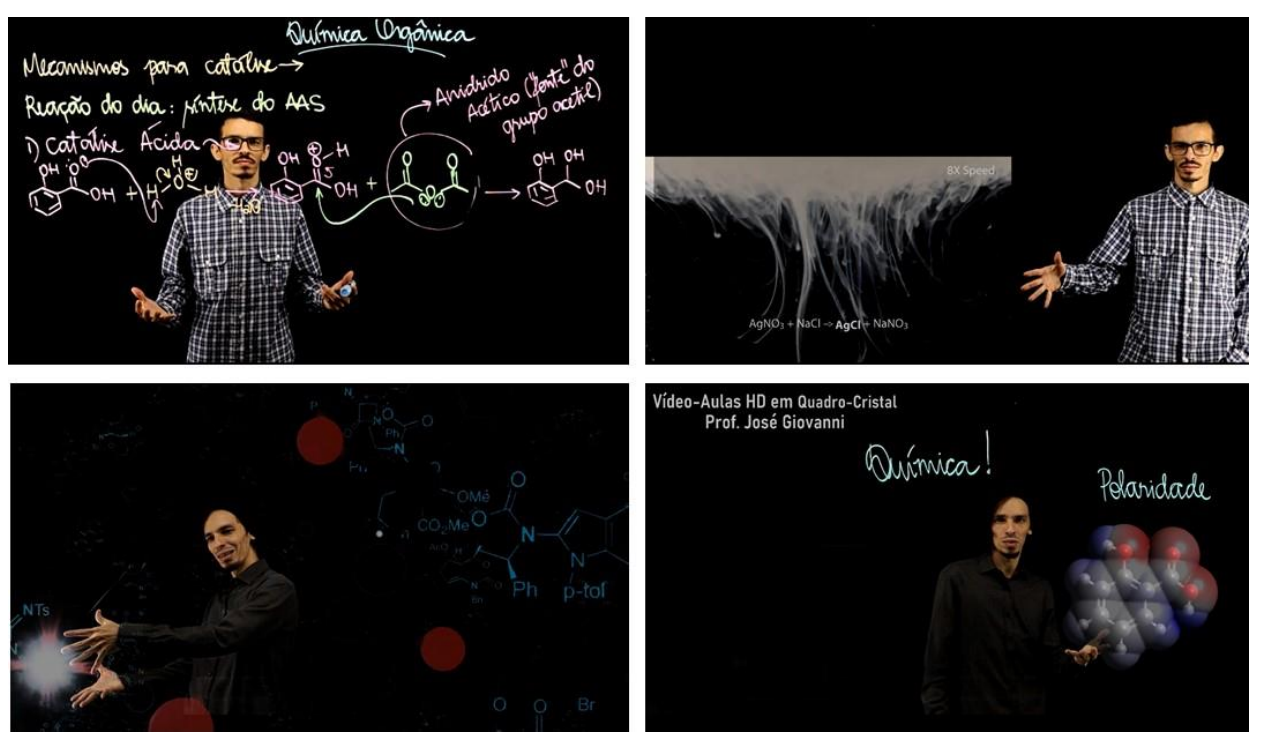

Figura 3 - O quadro-cristal em ação no Ensino de Química. Alto, em sentido horário: uso de canetas luminescentes para ilustrar mecanismos de reações orgânicas; uso de vídeo em aula sobre estequiometria; aula com superimposição de animações; apresentação de slides para discussão de polaridade.

\section{CONCLUSÕES E PERSPECTIVAS}

Neste artigo, foi descrita a experiência de construção do primeiro estúdio de quadro-cristal em uma instituição educacional pública do Distrito Federal. Contando com o apoio de um programa institucional e usufruindo de ferramentas gratuitas disponíveis na internet foi possível produzir conteúdo instrucional inovador, atraente, com logística reduzida, e sem a necessidade de longas e onerosas etapas de pós-produção.

Especialmente por conta do novo cenário de educação no Brasil, esperamos com este trabalho incrementar o engajamento discente em cursos profissionalizantes por meio da aplicação dos materiais instrucionais produzidos com a ferramenta lightboard.

${ }^{a}$ Ainda não existe uma tradução científica do termo lightboard para a Língua Portuguesa. A proposta aqui cunhada (quadro-cristal), até onde nossas pesquisas de literatura permitem inferir, trata-se da primeira versão brasileira para o termo.

\section{Referências Bibliográficas}

BERGMANN, J; SAMS, A. Sala de aula invertida: uma metodologia ativa de aprendizagem. Trad. Afonso Celso da Cunha Serra. 1. ed. Rio de Janeiro: LTC, 2016.

BISHOP, J.L.; VERLEGER, M.A. The Flipped Classroom: A Survey of the Research. In: 120th ASEE ANNUAL CONFERENCE \& EXPOSITION, 23-26 junho, 2013, Atlanta, Georgia. Anais. Atlanta: American Society for Engineering Education, 2013, ps. 23.1200.1 - 23.1200.18. 
FULTON, K. Upside down and inside out: flip your classroom to improve student learning. Learning and Leading with Technology, junho/julho 2012, 39 (8), ps. 12-17.

FUNG, F. M. Adopting Lightboard for a Chemistry Flipped Classroom to Improve Technology-Enhanced Videos for Better Learner Engagement. Journal of Chemical Education, 2017, 94, ps. 956-959.

GUO, P.J.; KIM, J.; RUBIN, R. How video production affects student engagement: an empirical study of MOOC videos. In: FIRST ACM CONFERENCE ON LEARNING SCALE, Atlanta, Georgia, 2014. Anais. Atlanta: ACM, 4-5 março, 2014, ps. $41-50$.

JUNIOR, J. B.B. Sala de Aula Invertida: Recomendações e Tecnologias Digitais para sua Implementação na Educação. Revista Novas Tecnologias na Educação V. 17 No 2, agosto, 2019.

MATEUS, A.L.M.L.; MIRANDA, M.F.; BRAGA, R.A. COLTEC TUBE. 2017. Canal de vídeo-aulas para o ensino técnico. Disponível em: 〈http://lightboard.info/>. Acesso em: 12 dez. 2020.

MCCORKLE, S.; WHITENER, P. The Lightboard: Expectations and Experiences. International Journal of Designs for Learning. 2020, Volume 11, Ed. 11, ps. 75 - 83.

MENEGHETTI, L.C.; OLIVEIRA, I.M.; TRINDADE, Y.T.; PAULETTI, R.M.O.; BITENCOURT JR., L. A. G. A utilização da técnica lightboard na gravação de videoaulas. In: $4^{\circ}$ Congresso de Graduação 2018. Anais. Universidade de São Paulo, PróReitoria de Graduação, p. 257.

PESHKIN, M. How To Build a Lightboard. Google Scholar. Disponível em: <http://lightboard.info/>. Acesso em : 12 dez. 2020.

PIENTA, N.J.A. "Flipped Classroom". Reality Check. Journal of Chemical Education. 2016, 93 (1), ps. 1-2.

RUIPEREZ-VALIENTE, J.A.; MUÑOZ-MERINO, P. J.; LEONY, D.; DELGADO KLOOS, C. ALASKA: A learning analytics extension for better understanding the learning process in the Khan Academy platform. Comput. Human Behav. 2015, 47, 139-148.

SANTOS, A.O.; SILVA, R.P.; ANDRADE, D.; LIMA, J.P.M. Dificuldades e motivações de aprendizagem em Química de alunos do ensino médio investigadas em ações do (PIBID/UFS/Química). Scientia Plena 9, 077204, 2013.

SCHWEIKER, S.S.; GRIGGS, B.K.; LEVONIS, S. M. Engaging Health Student in Learning Organic Chemistry Reaction Mechanisms Using Short and Snappy Lightboard Videos. Journal of Chemical Education 2020, 97 (10), ps. 3867-3871.

SEERY, M. K. ConfChem Conference on Flipped Classroom: Student Engagement with Flipped Chemistry Lectures. Journal of Chemical Education, 2015, 92 (9), ps. $1566-1567$. 